\title{
PRE-TRANSPLANTATION CARE - QUALITY AND EFFECTIVENESS OF THE NURSING PROCESS IN PATIENTS ON HEMODIALYSIS
}

\author{
Veselina Vasileva \\ Department of Nursing Care, Faculty of Public Health, Medical University of Varna
}

\begin{abstract}
INTRODUCTION: One of the most serious problem in the field of organ donation and transplantation is the lack of donors. Due to this reason, the length of time patients spend awaiting organ transplantation has a different duration. For patients included in the list of pending kidney there is just one alternative option - hemodialysis. Maintaining high quality and good performance of the nursing process in this period is essential for the patient, since every year of dialysis causes irreversible damage.

AIM: The purpose of this survey is to study the views of 50 patients on hemodialysis regarding the implementation of various forms of training to improve the quality of the care before transplantation.

MATERIALS AND METHODS: A total of 50 patients undergoing hemodialysis treatment from the Clinic of Hemodialysis at the St. Marina University Hospital, Varna were interviewed by using a questionnaire method during the month of July 2016. The data was processed statistically by Microsoft Excel 2013.

RESULTS AND CONCLUSIONS: The aim of the research focuses on exploring the attitudes and the views of the patients to improve and maintain good health in the period before renal transplantation.

The importance of the problem was confirmed by the fact that over half of the respondents rated the quality of transplant care as low. Over $2 / 3$ of the respondents had never attended courses or specialized training regarding treatment options. The need to raise awareness about developments in the field of transplantation leads to interest in various forms of training and is supported by a significant proportion of patients. The need for innovative training methods determines the leading role of the nurse.
\end{abstract}

Keywords: quality, nursing process, teaching, patient, hemodialysis

Address for correspondence:

Veselina Vasileva

Faculty of Public Health

Medical University of Varna

55 Marin Drinov St

9002 Varna

e-mail: veselina.vasileva@mu-varna.bg

\section{INTRODUCTION}

Renal transplantation is a relatively new method of treatment. The first successful kidney transplant in the world was performed in Boston, USA, in 1954 and in Bulgaria - in 1968. Kidney transplantation is the preferred method for optimal treatment of patients with advanced terminal renal failure. Transplantation prolongs survival and improves the quality of life of transplant recipients. It also reduces the cost of treatment due to the cost difference between dialysis and post-transplantation immunosuppressive therapy (1). 
Veselina Vasileva

One of the most serious problems in the field of organ donation and transplantation is the lack of donors (2). It is for this reason that the length of time these patients spend awaiting organ transplantation has a different duration. Maintaining high quality and good performance of the nursing process in this period is essential for the patient, as every year of dialysis causes irreversible consequences. The damage caused to the body as a result of hemodialysis treatment is a risk factor for transplant rejection in transplant possible patients $(3,4)$.

\section{AIM}

The purpose of this study was to explore the views of the 50 patients undergoing hemodialysis treatment on attitudes towards implementation of various forms of training to improve the quality of care before transplantation.

Tasks:

1. To explore the views of the patients undergoing hemodialysis treatment on the quality care prior to transplantation in Bulgaria by assessing the level of care.

2. To determine the level of communication between patients and hospital staff in the period before transplantation by assessment.

3. To establish the role and place of the nurse in the period before transplantation.

4. To identify possible options for improving the quality of care before transplantation.

5. To present the attitudes of respondents toward different and innovative forms of learning.

\section{MATERIALS AND METHODS}

The study includes 50 patients (17 of which are included in the waiting list for kidney transplants) undergoing hemodialysis treatment from the Clinic of Hemodialysis at the St. Marina University Hospital in Varna, in the month of July 2016. Attached is the analysis of literature, documentary and questionnaire method (direct individual anonymous poll).

Analysis of the primary sociological information was performed using Microsoft Excel 2013.

\section{RESULTS}

The focus of the research is on exploring the attitudes and views of patients to improve and maintain good health in the period before renal trans- plantation. The importance of the problem was confirmed by the fact that over half of the respondents determined the quality of transplantation care as low $(56.80 \%)$. Approximately $1 / 3$ of the respondents believe that the level of care is satisfactory (34.10\%). Only $9.10 \%$ of the patients were satisfied with the quality of services provided in that period and indicated that their level was high (Fig. 1).

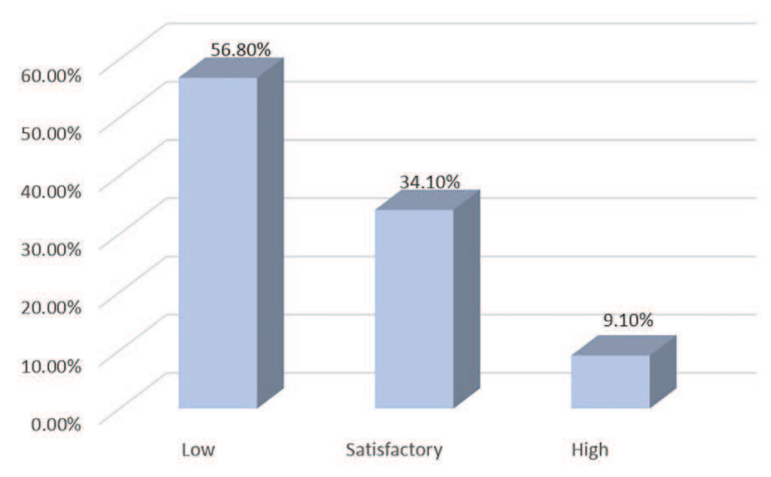

Fig. 1. Quality of the pre-transplantation care in Bulgaria

The lowest score given by respondents in terms of quality of care before transplantation requires a detailed study of their needs in order to optimize and increase the level of satisfaction among patients. The results presented in Fig. 2 reflect the attitudes of respondents to various proposals to improve the quality of life in the period prior to transplantation. The majority of the patients considered that maintaining higher standards in this period would help improve the quality of the healthcare (50\%). The proposal for organizing more courses and seminars on the topic gathered the support of 32.40 percent of the respondents. The next most popular response among respondents (11.80\%) was organizing support groups. Finally, $5.80 \%$ of patients believe that methods other than those listed in the effective methods should be used to meet their needs. None of them, however, indicated what they meant by useful (Fig. 2).

Supporting innovative practices in order to optimize the effectiveness of nursing process leads to improved quality of life for patients with chronic diseases. Some of these modern approaches have been taught in regular training sessions under various forms to patients and their relatives. This in turn raises the need to analyze the attitudes of patients to different forms of training aimed at improv- 
Pre-Transplantation Care - Quality and Effectiveness of the Nursing Process in Patients on Hemodialysis.

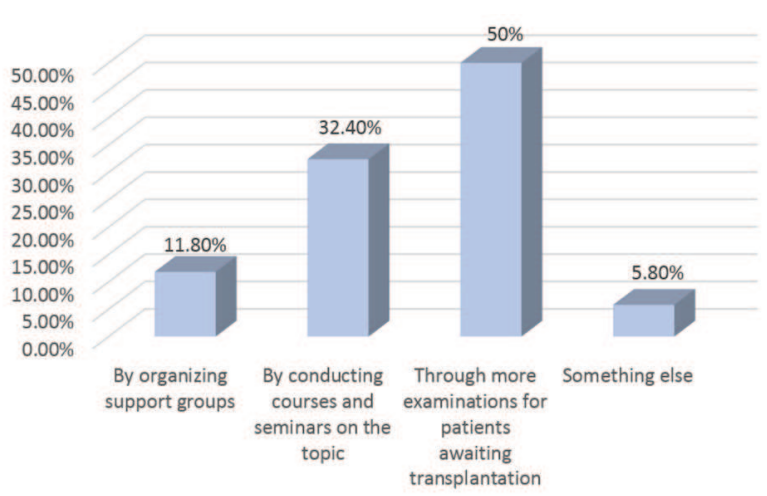

Fig. 2. Proposals to improve the quality of life during the pre-transplantation period

ing the quality of life before the transplantation period. Fig. 3 shows the attitude of the respondents to implementing specialized training on transplants and how to apply them, which was supported by 83.30 percent of them. Respondents believe that this form would be of particular benefit in this difficult period of their lives, both for themselves and for their families. A relatively low rate of $16.70 \%$ cannot assess the benefits of organizing such events featuring specialized training.

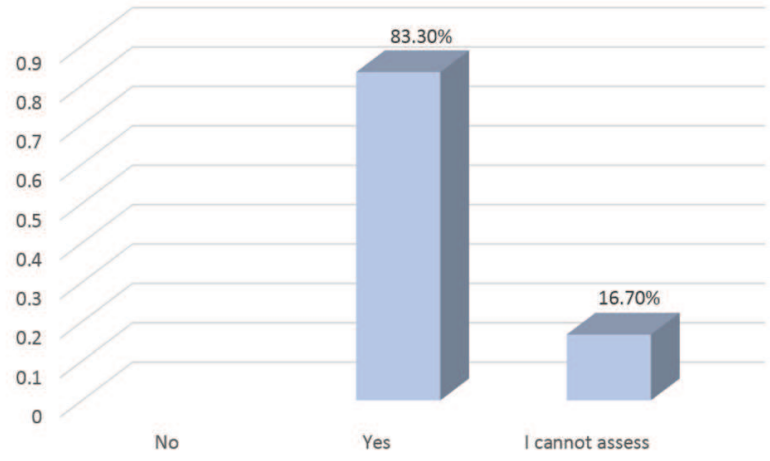

Fig. 3. Attitude to benefit from specialized training

Realizing the need and positive aspects of the organization of such form of education, a significant proportion of patients $(89.40 \%)$ think that it should be organized periodically to get acquainted with innovations in the field of transplantation and $65.20 \%$ add that it should be mandatory. The venue is an essential factor for attendance at such events as the majority of patients do not live in the city. As a logical consequence of these reasons, the majority of respondents (76.70\%) are willing similar training sessions to be carried out on the territory of the hemodialysis center. Of the respondents, $17.00 \%$ consider that these events should be organized in the transplantation center and only $6.40 \%$ support the idea of conducting specialized training at the Medical University of Varna.

With the dynamic development of nursing in recent years, there is an increasing need for more autonomy for the nurses. Introduction of innovative teaching methods creates favorable conditions for the development potential of the nurse. The idea of organizing various forms of training for nurses has the support of $93.30 \%$, and the remaining $6.70 \%$ express a negative attitude (Fig. 4).

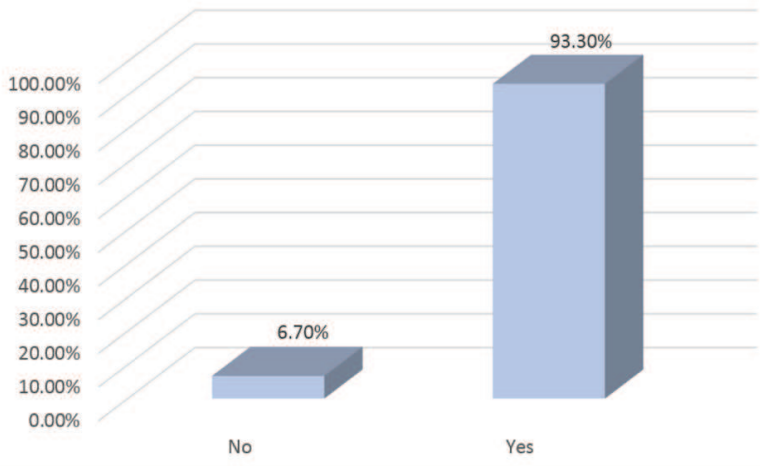

Fig. 4. Attitude towards conducting training of nurses

Another form of training on transplants and developments is training courses and seminars. The information presented there would be extremely useful for patients and their relatives prior to the transplantation period as they could get acquainted with both innovative practices and experiences of different countries. Over $90 \%$ (95.40\%) of respondents, however, had never attended courses or specialized training regarding treatment options. Only 4.60 percent said they have participated in such events (Fig. $5)$.

This alarming statistics requires the need for a thorough analysis among patients about the reasons for their decision. The leading factor among the majority of the respondents (82.10\%) is the lack of information on such events and $12.80 \%$ of those not attending gave reasons other than those mentioned. The remaining 5.10 percent presented financial issues as an argument for their decision. Respondents 
Veselina Vasileva

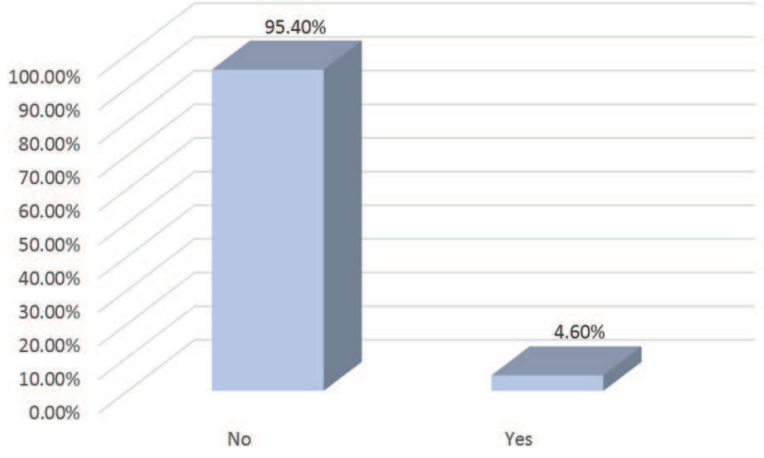

Fig. 5. Courses and seminars attendance on transplants and developments in the field

did not indicate the location of the event as a factor for not attending (Fig. 6).

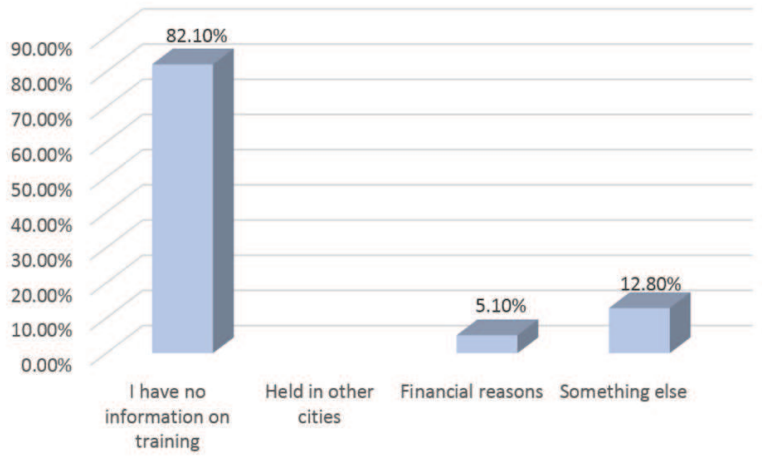

Fig. 6. Reasons for not attending courses and seminars on transplants and developments in the field

There is a need to raise awareness about developments in the field of transplantation and incorporated them into various and innovative forms of learning. Fig. 7 presents the attitude of the respondents to the online consultation with medical experts on transplants. The idea receives support among a

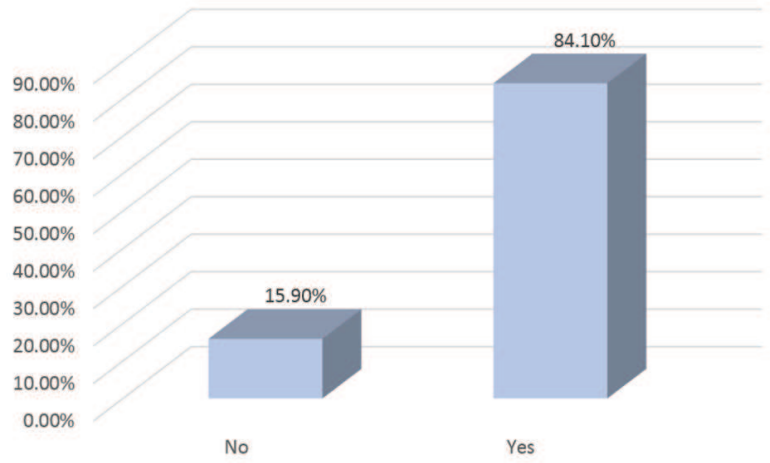

Fig. 7. Attitude towards organizing online consultation with medical experts on transplants significant proportion of the patients (84.10\%). Negative attitude towards the proposal is expressed by $15.90 \%$.

\section{DISCUSSION}

The subject is the attitudes and views of patients to improve and maintain good health in the period before renal transplantation. Wide survey of patients is one of the key elements that make up a sustainable system for assessing and monitoring the quality of care in this troubled period (5). The fact that over half of the respondents determine the quality of transplant care as low (56.80\%) is very important. The lowest score on the quality of care provokes a detailed study of the needs and their needs in order to optimize and increase the level of satisfaction among patients.

For the purposes of the study the opinion of respondents on various options for improving the quality of life before the transplant period was analyzed. The majority of patients considered having more check-ups in this period would help to improve the quality of care (50\%). Frequent examinations and consultations with various experts are instrumental in maintaining good health, which explains the leading result. The proposal for organizing more courses and seminars on the topic collected the support of 32.40 percent expressing considerable interest and provoking insightful analysis.

The object of study is the attitudes of patients to different forms of training aimed at improving the quality of life before the transplant period. The high positive support from $83.30 \%$ to implement specialized training on transplants and how to apply them in practice, requires special attention from the medical staff. In this difficult period of their lives, respondents considered that this form would be of particular benefit to themselves and their relatives. The role and place of relatives is often underestimated, but is of fundamental importance because they are an active participant both before the transplantation and during the nursing process (5). A significant proportion of patients $(89.40 \%)$ added that similar forms of training should be led periodically to get acquainted with innovations in the field of organ transplants, which must also become mandatory (65.20\%). The venue is an essential factor for attendance at similar events bearing in mind that the majority of patients 
Pre-Transplantation Care - Quality and Effectiveness of the Nursing Process in Patients on Hemodialysis.

do not live in the city, which is why most of the respondents $(76.70 \%)$ would like for them to be carried out on the territory of the hemodialysis center.

Nursing care has been developing very fast in the recent years. With the growing autonomy of the nurses arises also the need to develop their own potential. Introduction of innovative methods creates appropriate conditions for the development of medical professionals. Some of these modern approaches have been taught at regular training sessions in various forms to patients and their relatives. Conducting various forms of training for nurses meets the approval of 93.30 percent of the respondents. The high support shows that nurses enjoy greater trust and credibility among patients, which is a prerequisite for achieving good results after training.

Courses and seminars on transplants and developments in the field are another interesting form of education. The information from these forms would be extremely useful for patients and their relatives prior to the transplantation period as they get acquainted with innovative practices. An extremely high percentage $(95.40 \%)$ of respondents, however, had never attended courses or specialized training regarding treatment options.

This alarming statistics, focusing on the problem calls for a more thorough analysis among patients about the reasons for their decision. The majority of respondents indicated lack of information about such events as the main argument for not attendance (82.10\%). Here is the place to turn and look at the educational institutions. Training on transplants and treatment options for patients should be seen as a commitment not only of the medical teams, but also the educational institutions as part of their policy for health development and improving the quality of patient care.

The technological time in which we live provides a variety of innovative forms of learning. The idea of conducting online consultations with medical experts on transplants receives strong support from the patients $(84.10 \%)$. Facilities that provide advanced technologies save time and money.

\section{CONCLUSION}

There is a recognized need among patients for personal development and motivation to improve their knowledge and skills through visits of various forms of training. It involves organizing various forms of training, focusing on interactive methods of learning, discussion, lecture, case studies and role plays. It is better for training on transplants and treatment options for patients to be seen as a commitment not only of the medical teams, but also of educational institutions as part of their policy for health development and improving the quality of patient care.

Dynamically changing conditions, expectations and needs of modern society require mobility and flexibility to meet the interests of all participants - patients and medical professionals. Continuous learning and innovation contribute to the utilization of modern practices and approaches in the process of nursing care. All this in turn leads to a natural increase in the efficiency and quality of life of patients with chronic diseases.

\section{REFERENCES}

1. Georgieva S, Savova A, Paskalev E, Petrova D. Literary study of legislative requirements and drug therapy of renal transplant. General Medicine, Central Medical Library, MU Sofia.2012; 14 (3): 3-11. (in Bulgarian)

2. National campaign in support of donation [Internet]. Bulgaria; [ cited 2016 Aug 12]. Organ donation and transplantation; [about 2 screens]. Available from: http://www.mh.government.bg/bg/informaciya-za-grazhdani/donorstvo-i-transplantacii/ donori-i-nuzhdaeshi-se-ot-transplantaciya/

3. Meier-Kriesche HU, Kaplan B. Waiting time on dialysis as the strongest modifiable risk factor for renal transplant outcomes: A Paired Donor Kidney Analysis1 Transplantation. 2002;74(10):1377-81.

4. Meier-Kriesche HU, Port FK, Ojo AO, et al. Effect of waiting time on renal transplant outcome. Kidney Int. 2000; 58: 1311-7.

5. Muhina S, Tarnovska I. Theoretical foundations of nursing. In: Nursing process Pleven: Medical University Pleven Press; 2006. p. 89-115. (in Bulgarian) 